\title{
Aktuelle und künftige Herausforderungen für Führung aus den Umwelt- und Umfeldfaktoren des Unternehmens
}

Es wäre zu kurz gesprungen, Führungserfolg oder -misserfolg allein an der Person der Führungskraft festzumachen. Nähert man sich dem Thema Führung mit einem systemischen Blick, können die auf Führungskraft und Führungssystematik in einem Unternehmen einwirkenden Rahmenbedingungen nicht außer Acht gelassen werden. Die Geschichte zeigt, dass sich einzelne Führungsstile unter anderem am gesellschaftlichen und politischen Kontext oder, wie zu Zeiten der Industrialisierung, an einer rationalen, normativen und biologischen Perspektive orientiert haben.

Der Psychologe Kurt Lewin (1890-1947) hat mit seiner Feldtheorie die Bedeutung der Situationswahrnehmung und -bewertung durch das Individuum hervorgehoben. Lewin geht von der Grundannahme aus, Verhalten sei zielgerichtet und eine Funktion des für das Individuum zu einem bestimmten Zeitpunkt gegebenen „Lebensraumes“. Der Lebensraum umfasst sowohl die Person selbst als auch ihre Umgebung. Die Feldtheorie wird heute als allgemeine Rahmentheorie aufgefasst, mit deren Hilfe handlungs- und motivationspsychologische Fragestellungen ebenso bearbeitet werden können, wie etwa arbeits- und sozialpsychologische Fragestellungen (www.spektrum.de 2019).

Auch eine eigene repräsentative Studie mit über 800 Führungskräften im Bankenbereich zeigt, dass ein direkter Zusammenhang zwischen dem Führungsverhalten und der jeweiligen Situation und den Kontextfaktoren (Ressourcen, Strukturen, kulturellen Elementen einer Organisation) besteht (Burkhart 2012).

Bei zahlreichen Coaching- und Supervisionsgesprächen (Reflexionsgespräche in Führungsteams oder mit einzelnen Führungskräften zur Verbesserung des 
Führungsverhaltens) stoße ich in der Praxis regelmäßig auf Führungskräfte, die diesen Zusammenhang zum Thema machen. Immer wieder wird dabei deutlich, wie sehr aktuelle Umwelt- und Umfeldfaktoren, gerade in Zeiten des Umbruchs, die Führungssystematik des Hauses und damit das Führungsverhalten der Führungskräfte beeinflussen.

Insofern macht es Sinn, auf die wesentlichen Rahmenbedingungen kurz einzugehen.

\subsection{Wirtschaftliche und politische Rahmen bedingungen}

Banken und Sparkassen haben bereits bessere Rahmenbedingungen erlebt als die aktuell vorherrschenden. So sehen sie sich (Stand: Dezember 2019 und anhaltend im Frühjahr 2020) unter anderem

- dem möglichen Ende einer langen Wachstumsphase in Deutschland,

- einer nachhaltend angespannten wirtschaftlichen Situation in einigen Ländern Europas,

- den unklaren Folgen und möglichen Auswirkungen des Brexit, sowie

- aktuellen globalen Handelskonflikten und deren Auswirkungen auf die Weltwirtschaft

gegenüber (Wuermeling 2019). Hinzu kommen die wirtschaftlichen Auswirkungen unvorhersehbarer Ereignisse, wie z. B. die enormen Einbrüche der Aktienmärkte aufgrund des SARS-CoV-2 (Coronavirus) im Frühjahr 2020.

Die aufsichtsrechtlichen Anforderungen bleiben weiterhin hoch, auch wenn die Forderungen nach ausreichendem Eigenkapital und einer adäquaten Risikooptimierung zurzeit weitestgehend erfüllt werden können. Die Kapitalanforderungen (BASEL IV, TRIM, MaRisk) wirken ebenso einschränkend auf die Geschäftspolitik, wie die Anforderungen aus dem Kunden- und Datenschutz (MIFID II, DSGVO, PSD II).

Gleichzeitig nimmt der wirtschaftliche Druck weiter zu. Das Ergebnis im deutschen Privatkundengeschäft wird nach einem Rückgang um 62 \% von 2010-2017, bei einem gleichzeitigen Anstieg der Cost-Income-Ratio in diesem Segment von $82 \%$ auf $92 \%$ in den nächsten fünf Jahren weiter zurück gehen. Allein zur Stabilisierung des bestehenden Ertragsniveaus wären effektive Kostensenkungen von $16 \%$ erforderlich. Die Analysen einer zeb-Studie prognostizieren, dass z. B. Sparkassen bei einer anhaltenden Niedrigzinsphase und ohne kompensierende Maßnahmen im Jahr 2021 einen Nullgewinn ausweisen werden. Dies gefährdet die Rentabilität und Tragfähigkeit deren Geschäftsmodells (zeb 2018). 
Erstaunlich ist, dass sich viele Entscheider*innen heute von der wirtschaftlichen Entwicklung der vergangenen Jahre überrascht zeigen. So machen sie gerne die Weltwirtschaftskrise 2008 und ihre Folgen als einen Hauptgrund für den negativen Trend aus, wie er sich heute abzeichnet. Dabei deuteten bereits Mitte der 90erJahre viele Anzeichen darauf hin, dass eine dringende Anpassung der Geschäftspolitik erforderlich sein würde, wollte man sein Institut zukunftsfähig aufstellen (siehe Background Information).

Bereits 1994 weist die Priewasser-Prognose für den deutschen Bankenmarkt deutliche Rückgänge im Zinsüberschuss, eine Unwucht bei der Relation Zinsüberschuss zu Provisionsüberschuss mit eindeutigen Anzeichen für Sättigungsgrenzen im Provisionsüberschuss sowie anhaltend hohe Risikokosten mit Blick auf die kommenden 15 Jahre aus. Die Empfehlungen für die künftige Entwicklung der Geschäftsmodelle von Banken und Sparkassen waren: Kostensenkungsprogramme, vor allem im Personalbereich, eine intensive Nutzung der Standardisierungsmöglichkeiten, vor allem im Privatkundengeschäft, sowie das Eingehen strategischer Allianzen und strategischer Netzwerke (Priewasser 1994). Auch ohne die im Jahr 2008 beginnende Finanzkrise hätte somit dringend Handlungsnotwendigkeit bestanden, um der sich abzeichnenden Abschmelzung des Kundengeschäfts entgegenzuwirken. Die Finanzkrise und die bis heute anhaltenden Folgen daraus hatten daher eher die Funktion eines Brandbeschleunigers für einen sich bereits abzeichnenden Flächenbrand.

\section{Strukturwandel und Wettbewerb}

Die Entwicklung des Wettbewerbs im deutschen Bankensektor wird entscheidend davon geprägt werden, wie gut es neuen Technologien und Wettbewerbern gelingt, im Finanzsystem Fuß zu fassen und wie schnell die traditionellen Akteure im Finanzsystem neue Technologien adaptieren können. (Buch 2018)

Bezeichnend für die Dynamik am Markt neuer Technologien ist, dass einige der im Überblickswerk „Innovative Geschäftsmodelle im Banking“ (TME Institut für Vertrieb und Transformationsmanagement e.V. 2014) aufgeführten FinTechs heute nicht mehr, oder so nicht mehr existieren. Neue FinTechs entstehen, bestehende werden aufgekauft oder verschwinden aufgrund des Wettbewerbsdrucks komplett.

Zusätzlich zu FinTech-Unternehmen entwickeln die sogenannten BigTechs, wie Apple, Google, Amazon und Facebook auf ihren eigenen Technologieplattformen Produkte und Dienstleistungen, die im direkten Wettbewerb zu den traditionellen Anbietern im Finanzsystem stehen.

Ungeachtet konkreter Anbieter gibt die Deutsche Bundesbank einen Überblick zu technologischen Finanzinnovationen und deren Hauptanwendungsgebieten. Abb. 2.1 greift diese auf. 


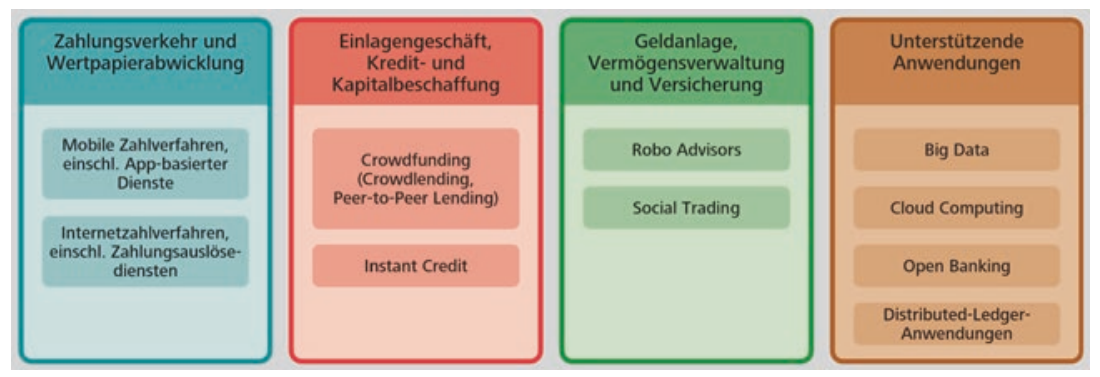

Abb. 2.1 Technologische Finanzinnovationen und Hauptanwendungsgebiete. (Copyright: Deutsche Bundesbank, Frankfurt am Main, Deutschland)

Diese Finanzinnovationen können den Wettbewerb in Teilen des Finanzsystems erhöhen und damit die Margen senken (z. B. durch Crowdlending oder auch Robo Advisor).

Die Auswirkungen dieser Innovationen auf die Struktur des Finanzsystems sind derzeit kaum final abschätzbar. Die technologischen Neuerungen leiten jedoch einen Strukturwandel im Finanzsektor ein.

Nach wie vor verspüren Finanzinstitute einen hohen wirtschaftlichen und regulatorischen Druck, wenn es um die dauerhafte Sicherung oder gar zukunftsorientierte Weiterentwicklung ihres Geschäftsmodells geht.

Aktuell ist beobachtbar, dass strategische Projekte konsequenter vorangetrieben werden. Dabei haben aufsichtsrechtliche Themen und Projekte nach wie vor Vorrang. Ebenso konsequent geht man, nicht zuletzt aufgrund der technologischen Möglichkeiten, die Standardisierung von Prozessen mit dem Ziel an, Effizienz und damit Kosteneinspareffekte zu erzielen.

Auf der Ebene des Managements ist der Spannungsbogen zwischen der Gewährleistung einer MaRisk-konformen Risikotragfähigkeit, einer notwendigen Ertragsstabilisierung, einer zukunftsgerichteten Entwicklung des Hauses und einer gleichzeitigen Kostenoptimierung deutlich spürbar.

Mit Blick darauf ist es zielführend, die einzelnen Teilbereiche der Bank/ Sparkasse strategisch miteinander zu verzahnen, die Wechselwirkungen der unterschiedlichen strategischen Ziele auf der Ebene des Managements zu diskutieren und daraus resultierend, eine abgestimmte Planung und Umsetzung im Gesamthaus zu koordinieren. Aufsichtsrechtlich wird dem unter anderem über die schlüssige Dokumentation der Risiko- und Geschäftsstrategie Rechnung getragen. 
In der Praxisumsetzung zeigen sich indes Unwuchten in der Steuerung des Gesamthauses. Insbesondere dann, wenn unterschiedliche Vorstellungen zu strategischen Zielrichtungen der Bank/Sparkasse unter Vorständen bestehen und diese nicht auf Augenhöhe ausdiskutiert und in eine gemeinsame Zielausrichtung überführt werden.

Der regulatorische und wirtschaftliche Druck kommt zunehmend auf der Mitarbeitendenseite an. Sei es, wenn die umfangreichen Auflagen der Beratungsdokumentation im Kundengespräch umgesetzt werden müssen oder, wenn Kostensenkungsmaßnahmen über Einsparungen bei den Personalkapazitäten realisiert werden.

\section{Praxisbeispiel}

Ein in der Praxis beobachtbarer nachteiliger Effekt entsteht durch das an Benchmarks orientierte Einsparen von Personalkapazitäten. Diese Benchmarkwerte orientieren sich an den besten Häusern der vergleichbaren Bankengruppe. Mit Blick auf diese Werte spart das Finanzinstitut in einigen Fällen in Vorausschau auf gerade erfolgte oder künftig geplante Prozessoptimierungen bereits Personalkapazitäten ein, obwohl die Effizienz in Form von Arbeitsentlastung durch verschlankte Prozesse auf der Arbeitsebene längst noch nicht ankommen ist. Dadurch entsteht ein Überforderungs- bzw. Überlastungsgefühl bei den Mitarbeiter*innen. Sie müssen die gleiche Menge an Arbeit mit gleicher Qualität, aber weniger Personal umsetzen. Dauert dieser Zustand länger an, führt dies nicht selten zu Unzufriedenheit und mit zunehmender Häufigkeit zu krankheitsbedingten Ausfällen.

Im Coaching suchen die betroffenen Fachführungskräfte bzw. Teamleiter*innen nach Lösungen für diese Situation. Ein ,die Mitarbeiter*innen bei der Stange halten“ greift in vielen Fällen längst nicht mehr. Der Ansatz, die Engpasssituation mit der darüber geordneten Führungskraft zu besprechen, scheitert oft daran, dass diese sich wiederum, oft in vorauseilendem Gehorsam, mit dieser Problematik beim Vorstand nicht positionieren will, um ihrer eigenen Reputation nicht zu schaden. Oder sie unternimmt einen Versuch, ihr zuständiger Ressortvorstand kann sich jedoch im Gesamtvorstand nicht durchsetzen.

Auch die fehlende Abstimmung im Gesamtvorstand ist regelmäßig Thema im Coaching der zweiten Führungsebene, vor allem bei Führungskräften mit Vertriebsverantwortung. Dominiert zum Beispiel der Steuerungsvorstand den Gesamtvorstand, kann dies zu einer übersteigerten Regulatorik im Gesamthaus führen. Die Arbeitsanweisungen und Regelungen, die dann von den Mitarbeiter*innen umzusetzen sind, gehen über ein gefordertes Normalmaß hinaus. Die Folge für 
den Marktbereich: Es wird zusätzliche Bearbeitungszeit gebunden, die dann die eigentliche Zeit mit dem Kunden/der Kundin schmälert. In Folge dessen entsteht ein "Stellvertreterkrieg“ auf der Ebene direkt unterhalb des Vorstandes, der bis auf die operative Ebene der Mitarbeiter*innen ausgetragen wird

\section{Fazit aus diesem Abschnitt}

Regulatorische Anforderungen und der anhaltende wirtschaftliche Druck auf die Banken und Sparkassen dürfen den Blick auf die Menschen in den Häusern nicht in der Art verändern, dass diese als Kostenstellen oder Plankapazitäten gesehen werden. Eine chancenorientierte Kommunikation ist einer angstschürenden Auseinandersetzung vorzuziehen, wenn es darum geht, Mitarbeiter*innen Mut für die anstehenden Veränderungen zu machen.

\subsection{Demografische Entwicklung und Management der Generationen}

\section{Demografische Entwicklung}

Es fehlt an allen Ecken und Enden an jungen Nachwuchskräften. Vor allem am Markt fehlen dringend erfahrene Berater*innen. Aufgrund des Eintretens geburtenschwacher Jahrgänge in den Arbeitsmarkt wird dieser Mangel noch verschärft, wenn die Generation der Baby-Boomer in den Ruhestand eintritt. So wird es auch in den kommenden Jahren schwierig bleiben, geeignete Fachkräfte zu rekrutieren.

Heute sind die Folgen der demografischen Entwicklung längst in der Bankenlandschaft angekommen.

Die Helmut-Schmidt-Universität in Hamburg benennt die Risiken für Banken und Sparkassen ohne ein Management der demografischen Entwicklung wie folgt:

- Verlust von (implizitem) Wissen

- Mangel an erfahrenen Mitarbeiter*innen und an qualifizierten Nachwuchskräften

- Anstieg altersbedingter Fehlzeiten

- Geringere Zufriedenheit wegen nicht-altersgerechter Führung

- Geringere Bindung wegen fehlender Entwicklungsmöglichkeiten

- Reduzierte Leistung bei Innovationsentwicklung, Produktivitätssteigerung und Veränderungsprozessen 
Als Folge dieser Risiken ergibt sich eine reduzierte Wettbewerbsfähigkeit (Felfe 2012).

Führungskräfte erleben in der Praxis gleich in zweierlei Hinsicht eine Herausforderung: Einerseits müssen sie sicherstellen, insbesondere das Erfahrungswissen der reifen Mitarbeiter*innen im Haus zu halten und im Idealfall frühzeitig auf nachfolgende zu übertragen. Andererseits müssen die Kompetenzen der jüngeren Mitarbeiter*innen schnell entwickelt und ihr Erfahrungswissen zügig aufgebaut werden.

Beide Aspekte sind zeitaufwendig und erfordern eine große Nähe zu den jeweiligen Mitarbeiter*innen. Durch die zunehmende Größe der Häuser und die damit verbundenen räumlichen Distanzen, die eine Führungskraft häufig überbrücken muss, um alle ihr zugeordneten Mitarbeiter*innen an den unterschiedlichen Standorten zu führen, ist diese Nähe oft nicht ausreichend leistbar.

Abb. 2.2 greift einzelne Stufen auf, in denen Menschen lernen bzw. Kompetenzen aufbauen. Je nach Kompetenzstufe sind unterschiedliche Aufgaben von Führung erforderlich. Gerade bei den Stufen der „unbewussten Inkompetenz“ und „,bewussten Inkompetenz", die immer dann auftreten, wenn Neuerungen erlernt, verinnerlicht und angewendet werden müssen, braucht es eine hohe Betreuungsintensität durch die Führungskraft. Sei es, um ihnen die Sorgen und Ängste vor anstehenden Herausforderungen zu nehmen, sie bei einem engagierten Schritt in Richtung Neuerung zu

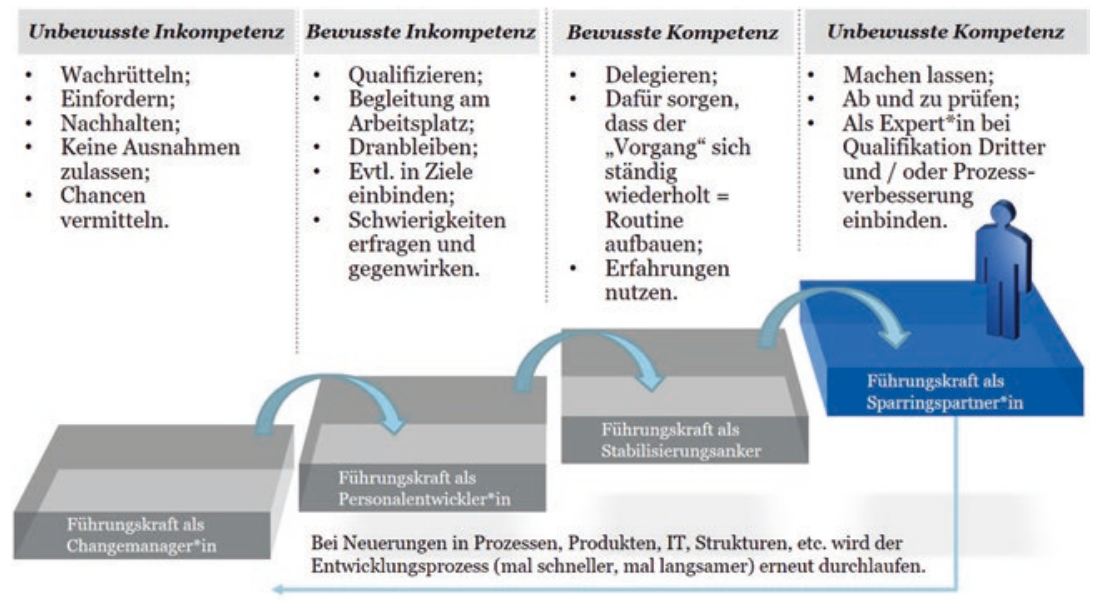

Abb. 2.2 Lern- und Entwicklungsstufen von Menschen und Aufgaben für Führung 
stützen oder, um sie mit gezielten Personalentwicklungsmaßnahmen am Arbeitsplatz zu begleiten (z. B. durch Training on the Job, Coaching).

Bei den reifen Mitarbeiter*innen gilt, das auf der Stufe der „,bewussten Kompetenz" vorhandene Wissen und die damit verbundenen Fähigkeiten zu vertiefen und in Routine zu überführen. Die Delegation von Aufgaben und die Anreicherung des Verantwortungsbereichs tragen hierzu bei. Das auf der Ebene der „unbewussten Kompetenz" vorhandene implizite Wissen gilt es, auch für andere zu sichern (z. B. durch Mentoring, altersgemischte Lern- und Arbeitstandems).

Der Führungskraft muss ein Spagat zwischen notwendiger Nähe und fehlender Zeit, bei teilweise zu hohen Führungsspannen und großen räumlichen Distanzen, gelingen. Schaut man sich die Häufigkeiten an, die Teamleiter*innen mit dem Coaching ihrer Mitarbeiter*innen tatsächlich verbringen, so zeigen sich hier große Defizite. Dass Mitarbeiter*innen die erforderlichen Entwicklungsschritte daher nicht oder zu langsam machen, ist nicht verwunderlich.

\section{Praxisbeispiel}

Speziell mit der Übertragung des Wissens von erfahrenen Mitarbeiter*innen, die das Haus (aus Gründen des Ruhestands oder der Umorientierung) verlassen, tut sich das ein oder andere Finanzinstitut schwer. Häufig haben Schlüsselpersonen bereits die Bank/Sparkasse verlassen, wenn ihre Nachfolger*innen die Funktion übernehmen. Selbst wenn eine gewisse Zeit des Übergangs möglich ist, weil z. B. der Nachfolger/die Nachfolgerin in der gleichen Abteilung arbeitet, gelingt selten eine systematische Übergabe des Wissens, vor allem des Anteils, der nicht in Arbeitsanweisungen und Stellenbeschreibungen nachgelesen werden kann: des impliziten Wissens oder auch Erfahrungswissens.

Implizites Wissen stellt den Teil des Wissens eines Individuums dar, den es sich im Laufe seiner Entwicklung durch Erfahrungen angeeignet hat. Dieser Teil des Wissens ist dem Individuum häufig nicht bewusst präsent und lässt sich - wenn überhaupt - nur recht mühsam in Worte fassen. Dies hat zur Folge, dass sich implizites Wissen nicht ohne weiteres übertragen lässt.

Um der Wissensabwanderung im Haus vorzubeugen, hat ein Finanzinstitut den systematischen Wissenstransfer mittels einer Wissensstafette eingeführt und umgesetzt. Dabei steht der direkte Austausch zwischen den Menschen im Vordergrund. Drei Zielfelder standen im Mittelpunkt:

- Führungswechsel

- Reibungslose und effiziente Wechsel gestalten.

- Dialog zwischen Vorgänger*in und Nachfolger*in und die Integration der Mitarbeiter*innen in den Prozess stehen im Vordergrund. 
- Fachwechsel

- Expertenwissen sichern und auf Nachfolger*innen bzw. Organisationseinheiten übertragen.

- Prozessorientiertes Vorgehen mit Methoden der Stafette.

- Projekt-Debriefing

- Expert*innenwissen sichern und auf Nachfolger*innen bzw. Organisationseinheiten übertragen.

- Prozessorientiertes Vorgehen mit Methoden der Stafette.

Die nachfolgenden Fragestellungen helfen, um sich einen Überblick zur Ausgangssituation zu verschaffen:

- Wer wird in absehbarer Zeit das Team verlassen und wertvolles Wissen mitnehmen?

- Welche anderen Personen besitzen erfolgskritisches Wissen für die Zukunft? Wer würde noch von diesem Wissen profitieren?

- Wie viel Wissenstransfer und Informationsaustausch sind in meinem Team erforderlich?

- Kommt der Wissensaustausch zwischen den Altersgruppen von selbst in Gang oder muss ich diesen bewusst fördern?

Am Beispiel eines Führungswechsels soll im Folgenden die Vorgehensweise des Finanzinstituts in der Praxis vorgestellt werden.

Die Wissensstafette für den Führungswechsel ist eine Methode zum Transfer des Erfahrungswissens von ausscheidenden oder wechselnden Führungskräften auf deren Nachfolger*innen. In halbstrukturierten Interviews wird das personengebundene Wissen erhoben und in moderierten Übergabegesprächen zwischen Vorgänger*in und Nachfolger*in transferiert.

Im Regelfall startet bei den Führungskräften, die bis zu ihrem Ausscheiden kürzer (bei Kündigung) oder gleich einem Jahr (anlässlich des bevorstehenden Ruhestandes) bei dem Finanzinstitut arbeiten, automatisch der Prozess der Wissensstafette. Der Prozess wird von einem/einer neutralen Experten/Expertin, dem/der internen Bildungspartner*in, moderiert.

Die Wissensstafette gibt Orientierung und Struktur bei der Übernahme einer neuen Position, indem systematisch Hintergrund- und Erfahrungswissen erarbeitet werden. Die strukturierte Vorgehensweise und die kompetente Begleitung durch den/die interne(n) Personal Business Partner*in (Bildungspartner*in) als Moderator*in, tragen wesentlich zum schnellen und erfolgreichen Umstieg in die neue Führungsaufgabe bei. Zwei Aspekte stehen im Vordergrund: Zum einen der persönliche Dialog zwischen Vorgänger*in und Nachfolger*in, zum anderen die 
gegenseitige Erwartungs- und Zielklärung zwischen der neuen Führungskraft und ihrem/ihrer Vorgesetzten. Bei bestimmten Teilabschnitten im Prozess können darüber hinaus die Mitarbeiter*innen integriert werden.

In den Übergabegesprächen werden die Aufgaben- und Wissensgebiete des Wissensgebers/der Wissensgeberin strukturiert und in einer Mindmap visualisiert. Der Wissensnehmer/die Wissensnehmerin kann dabei eige Wissensbedürfnisse einbringen, die dann unmittelbar ihren Niederschlag in der Mindmap finden. Diese Mindmap kann als eine Art Wissenslandkarte angesehen werden.

Abb. 2.3 zeigt die Grundstruktur der Wissenslandkarte, die die Ausgangsbasis für das Gespräch bildet.

Um ein Gefühl für die weitere Vorgehensweise zu vermitteln, werden nachfolgend exemplarisch einige Fragen zu drei Teilfeldern der Wissenslandkarte aufgeführt, die als Leitfragen für die Gesprächsführung dienen:

\section{Kulturwissen}

- Wie würden Sie einem Außenstehenden die Kultur des Hauses/des Bereichs/ der Abteilung beschreiben?

- Woran lässt sich das festmachen (Beispiele)?

- Wie würde der Vorstand/die unmittelbare Führungskraft dies beschreiben?

- Was sind geheime Spielregeln, Do's und Dont's, die in keinem Schriftstück geregelt sind?

Beziehungsnetzwerk

- Welche Arbeitskreise, Erfahrungsrunden gibt es? Welchen Nutzen bringen sie für die eigene Arbeit?

- Wo sollte man aus politischen, strategischen oder sonstigen Gründen unbedingt anwesend sein?

- Welche kann man delegieren?

- Welche Ansprechpartner*innen/Expert*innen aus den benachbarten Abteilungen gibt es?

- Was können diese Expert*innen zusätzlich an Wissen beitragen?

- In welcher Weise beeinflussen die Schnittstellen die eigene Arbeit?

- Gibt es Schnittstellenmeetings?

- Gibt es Überschneidungen/Ergänzungen?

- Welche Absprachen gibt es?

- Gibt es konkurrierende Situationen und wie geht man damit um?

- Welche externen Kontakte sind wichtig, welche sind weniger wichtig? 


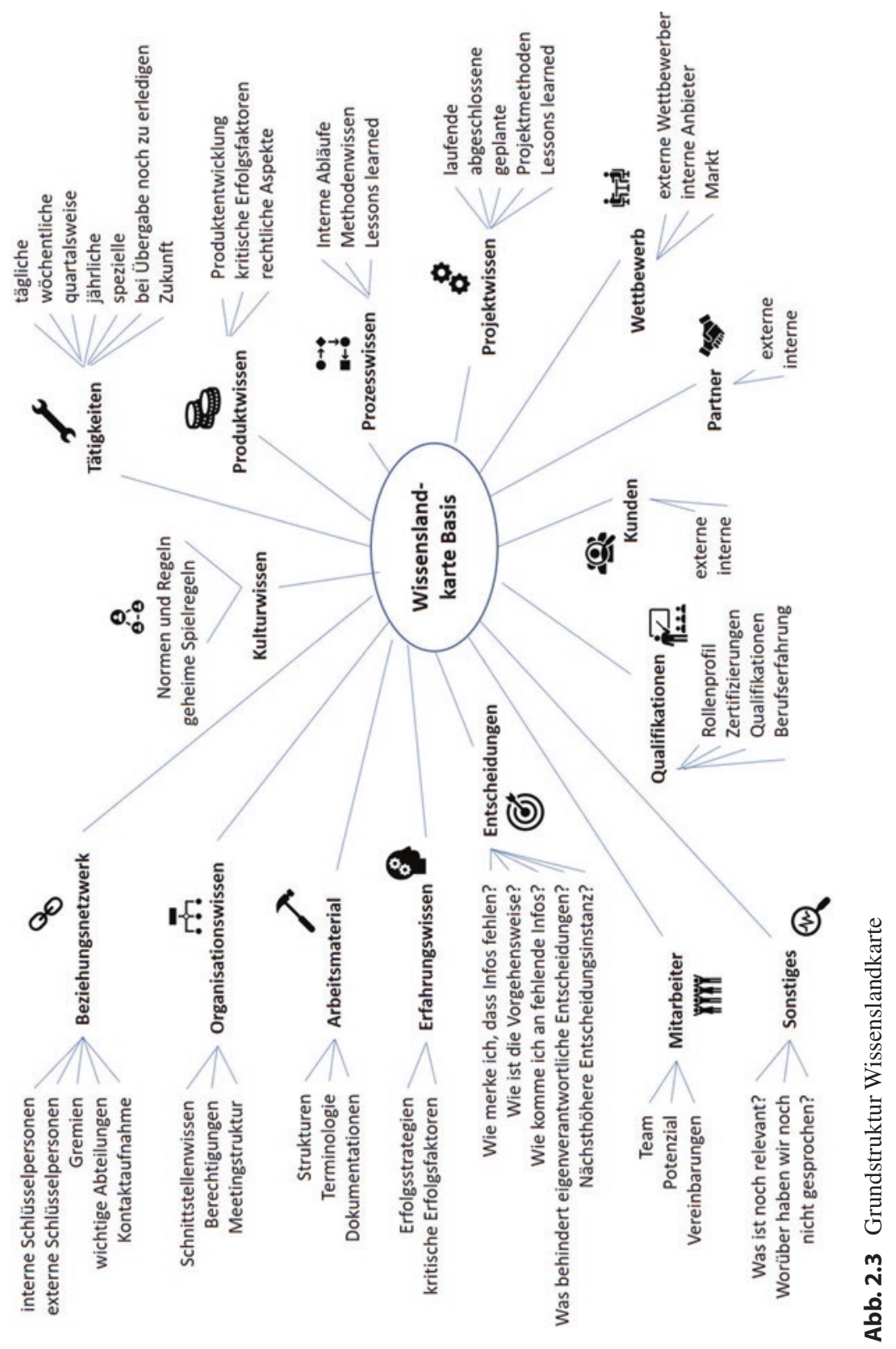




\section{Erfahrungswissen}

- Was lässt sich aus Ihrer Erfahrung nicht einfach so „lernen“?

- Welchen Anteil haben Erfahrungswissen und Fachwissen zueinander (in \%)?

- Was hat sich aus Ihrem Erfahrungsschatz bewährt, um dieses Know-how aufzubauen?

- Wie ist es Ihnen gelungen, Fachwissen mit Erfahrungswissen zu verknüpfen?

- Welche Ratschläge würden Sie aus der Brille des/der erfahrenen Hasens/ Häsin einem Neuling geben?

- Welche Stolpersteine gab es und wie sind Sie damit umgegangen?

- Was machen Sie, wenn Sie mal nicht mehr weiterwissen?

Das Gespräch läuft oft in Interviewform ab. Dabei stehen unter anderem Arbeitsalltag, Ergebnisse und besondere Erfolge bzw. Misserfolge im Zentrum.

Es wird ein Aktionsplan formuliert, mit der verantwortlichen Führungskraft abgestimmt und der Ablauf und Verantwortlichkeiten genau festlegt (welche Themen sollen wie/wann/durch wen vermittelt werden?).

Damit es nicht nur beim impliziten Wissenstransfer bleibt, entstehen aus diesen Gesprächen oft ergänzende Dokumente, wie z. B. Prozessbeschreibungen, Checklisten, Handbücher oder sogar Audio-Aufzeichnungen.

Kann ein Sechs-Augengespräch nicht realisiert werden, da der Nachfolger/die Nachfolgerin z. B. extern besetzt wird und erst nach Ausscheiden der Führungskraft die Stelle antritt, erfolgen die Gespräche zeitlich versetzt (Führungskraft mit Bildungspartner*in, anschließend Bildungspartner*in mit Nachfolger*in).

\section{Management der Generationen}

Gerade in Zeiten großen Umbruchs nehmen die Spannungen in altersgemischten Teams tendenziell zu: Die Arbeitsgeschwindigkeiten sind teilweise unterschiedlich. Der dabei erforderliche Einsatz technologischer Tools erfolgt in unterschiedlichen Graden. Neuerungen werden jeweils unterschiedlich schnell auf die eigene Arbeit adaptiert und Einzelne können mit der Veränderungsgeschwindigkeit unterschiedlich schnell Schritt halten.

Einzelnen Generationen lassen sich Tendenzen in ihren Neigungen, Werten, Einstellungen oder beruflichen Präferenzen zuschreiben.

Die Tab. 2.1 gibt einen Überblick zu den Generationen und den sie prägenden Einflüssen. 
Tab. 2.1 Zeitliche und sachliche Abgrenzung der Generationen (Krüger 2016)

\begin{tabular}{l|l|l|l}
\hline & Geburtszeitraum & Prägende Einflüsse & $\begin{array}{l}\text { Heutiges } \\
\text { Alter }\end{array}$ \\
\hline $\begin{array}{l}\text { Generation } \\
\text { Babyboomer }\end{array}$ & $1946-1964$ & $\begin{array}{l}\text { Aufarbeitung von 2. Weltkrieg und } \\
\text { Kriegsfolgen, Wirtschaftsaufschwung/ } \\
\text {,Wirtschaftswunder“ }\end{array}$ & $\begin{array}{l}\text { 56-74 } \\
\text { Jahre }\end{array}$ \\
\hline Generation X & $1965-1979$ & $\begin{array}{l}\text { Individualisierung, Bildung von } \\
\text { Subkulturen (Jugend/Studenten) und } \\
\text { Protestbewegung, erste Ölkrise/ } \\
\text { „Ölpreisschock“, beginnender } \\
\text { demografischer Wandel (Antibabypille) }\end{array}$ & $\begin{array}{l}\text { J1-55 } \\
\text { Jahre }\end{array}$ \\
\hline Generation Y & $\begin{array}{l}\text { 1980-1994 (bis } \\
\text { 2000) }\end{array}$ & $\begin{array}{l}\text { Einzug des Computerzeitalters in fast } \\
\text { sämtliche Lebensbereiche/Digitalisierung }\end{array}$ & $\begin{array}{l}\text { 20-40 } \\
\text { Jahre }\end{array}$ \\
\hline Generation Z & $\begin{array}{l}\text { ab 1995 (ab } \\
\text { 2001) }\end{array}$ & $\begin{array}{l}\text { Wirtschaftliche Unsicherheit/Finanzkrise, } \\
\text { „Nine Eleven“ und internationale Folgen }\end{array}$ & $\begin{array}{l}\text { 19 Jahre } \\
\text { und } \\
\text { jünger }\end{array}$ \\
\hline
\end{tabular}

Doch wie wirkt sich dies auf ihre Präferenzen mit Blick auf die Arbeitswelt aus?

Tab. 2.2 liefert einige Antworten hierauf.

Die Employer Branding Agentur Junges Herz (2019) verweist im Vergleich zu den Vorgängergenerationen auf die veränderten Werte der Generation Z. Dazu gehören:

- Wunsch nach freier Entfaltung und dennoch unbefristete Verträge.

- Große Sorgen in Bezug auf die eigene Zukunft.

- Immer weniger Identifikation mit bekannten Managern wie Martin Winterkorn, Tim Cook oder Bill Gates.

- Wunsch nach Flexibilität und Wechselhaftigkeit in der Arbeitswelt.

- Schlagworte wie Work-Life-Balance oder Familienfreundlichkeit finden immer weniger Gehör, da sie von vielen Arbeitgebern meist nur versprochen, jedoch nicht eingehalten werden.

- Führungsverantwortung wird zunehmend unattraktiver. Freizeit spielt eine viel größerer Rolle.

- Die Arbeitgebermarke gewinnt immer mehr an Bedeutung, da erlerntes Verhalten (Bewertungen im Internet, Tests, Vergleiche, Rankings) immer wichtiger wird.

- Die Digitalisierung gehört zwingend in den Berufsalltag. Social Media Verbot in der Ausbildung? Ein No-Go!

- Arbeitsmarktforscher stellen das Konzept der festen Arbeitsplatzzuweisung vermehrt in Frage. Home-Office und Office-Sharing sind keine Mode-Trends. 
Tab. 2.2 Postulierte Generationspräferenzen hinsichtlich der Arbeitswerte und Mitarbeiterbindungsmaßnahmen (Hülsbeck und Scheren 2017)

\begin{tabular}{|c|c|c|c|}
\hline $\begin{array}{l}\text { Arbeitswerte/ } \\
\text { HRM-Methode }\end{array}$ & Babyboomer & Generation X & Generation Y \\
\hline Arbeitswerte & \multicolumn{2}{|c|}{$\begin{array}{l}\text { Entscheidungsgewalt über andere } \\
\text { Menschen haben; } \\
\text { Ein prestigeträchtiger, hoch angesehener } \\
\text { Job; } \\
\text { Ein gutes Gehalt und gute } \\
\text { Arbeitsbedingungen; } \\
\text { Beschäftigungssicherheit. }\end{array}$} & $\begin{array}{l}\text { Eine interessante und } \\
\text { abwechslungsreiche } \\
\text { Arbeit; } \\
\text { Einen Beitrag für die } \\
\text { Menschen und die } \\
\text { Gesellschaft leisten; } \\
\text { Mit Menschen } \\
\text { arbeiten; } \\
\text { Sozialer Kontakt mit } \\
\text { den Kollegen. }\end{array}$ \\
\hline \multirow[t]{2}{*}{ Mitarbeiterbindung } & \multicolumn{2}{|l|}{$\begin{array}{l}\text { Gehalt; } \\
\text { Tantieme; } \\
\text { Aktienoptionen; } \\
\text { Altersvorsorge. }\end{array}$} & $\begin{array}{l}\text { Flache Hierarchien; } \\
\text { Unternehmenskultur; } \\
\text { Wertschätzung; } \\
\text { Lob; }\end{array}$ \\
\hline & $\begin{array}{l}\text { Flache Hierarchien; } \\
\text { Unternehmenskultur; } \\
\text { Wertschätzung; } \\
\text { Lob. }\end{array}$ & $\begin{array}{l}\text { Flexible } \\
\text { Arbeitszeitmodelle. }\end{array}$ & $\begin{array}{l}\text { Integre Arbeitsweise; } \\
\text { Individuelle } \\
\text { Arbeitsaufgaben; } \\
\text { Herausfordernde } \\
\text { Arbeit. }\end{array}$ \\
\hline
\end{tabular}

Wenn es um das Miteinander von Menschen geht, ist das Denken in Schubladen grundsätzlich nicht förderlich. So ticken sicherlich nicht alle Vertreter*innen einer bestimmten Generation gleich. Wer zum Beispiel zwischen 1946 und 1964 geboren ist und somit der Generation Babyboomer (oder auch Digital Immigrants) ,zugeordnet" wird, kann sehr wohl eine sehr hohe Affinität und Kompetenz im Umgang mit neuen Technologien vorweisen. Dies ist unter anderem durch Persönlichkeitsmerkmale bedingt, worauf in Kap. 5 noch näher eingegangen wird.

Führungskräfte versuchen die Unterschiedlichkeiten im Team in der Praxis in Teamsitzungen durch für alle gleichsam geltende Anweisungen und Maßnahmen auszubalancieren. Was als erster Schritt hilfreich ist, kann seine komplette Wirkung oft nicht entfalten, da die Gründe für Leistungsunregelmäßigkeiten oder Unstimmigkeiten im Team individuell sehr unterschiedlich sein können.

Ein weiteres Spannungsfeld beim Management unterschiedlicher Generationen liegt darin, dass junge Nachwuchskräfte mit zunehmender Ungeduld schneller von Aufstiegsmöglichkeiten und/oder Lohnsteigerungen profitieren wollen. Führungskräfte können jedoch aufgrund der restriktiven Gehalts- und Personalentwicklungspolitik einzelner Häuser nur unzureichend auf diese Forderungen eingehen. Und nicht immer ist dies auch opportun, da sonst die Spannbreite an personalpoli- 
tischen Maßnahmen je Mitarbeiter*in zu schnell ausgereizt wird. Die Gefahr steigt, dass die jungen Potenzialträger*innen das Haus verlassen.

Gehen sie wiederum auf diese Forderungen ein, verspüren ältere Mitarbeiter*innen eine Ungerechtigkeit. Nach ihrem Empfinden verdienen die jüngeren Kolleg*innen zu schnell zu viel bzw. erhalten früher Entwicklungsmöglichkeiten, als dies bei ihnen in der Vergangenheit der Fall war.

Im Extremfall setzen Führungskräfte ausschließlich auf die jüngeren Mitarbeiter*innen im Team, weil diese mit den anstehenden Veränderungen schneller Schritt halten können. Bei den reiferen Mitarbeiter*innen entsteht dabei oft das Gefühl, auf dem Abstellgleis geparkt zu werden.

\section{Fazit aus diesem Abschnitt}

Die Erwartungen unterschiedlicher Generationen an die Arbeitswelt machen Führungskräfte zunehmend zu Vermittlern zwischen den unter Umständen divergierenden Werte- und Arbeitsvorstellungen ihrer Mitarbeiter*innen. Unabhängig der Einordnung von Mitarbeiter*innen in einzelne Generationengruppen ist es ratsam, einzeln auf die aktuelle Situation und die künftigen beruflichen Vorstellungen je Mitarbeiter*in einzugehen. So wird Führung höchst individuell und holt den Einzelnen bei seinen Wahrnehmungen, Einstellungen und Wertvorstellungen ab. Hierzu bedarf es einer Nähe zum Mitarbeiter/zur Mitarbeiterin und einem angemessenen Pensum an zeitlichen Ressourcen.

\subsection{Kundenverhalten und Wertewelten}

\section{Kundenkommunikation wird digitaler}

Exemplarisch für die zahlreichen Untersuchungen zum veränderten Kundenverhalten wird auf die Studie von 2b AHEAD eingegangen. Die Trendstudie untersucht mit dem Blick auf das Jahr 2025 die Auswirkungen der Digitalisierung auf den Dialog zwischen Kund*innen und Unternehmen und somit ein Kernelement in der Kunde-Bank-Beziehung (2b AHEAD ThinkTank 2018).

Die folgenden acht zentralen Erkenntnisse ...

- Kund*innen suchen eine maximale Individualisierung, bei eigenem minimalen Aufwand. 
- Kund*innen erwarten Individualität, Menschlichkeit, Einfachheit, Geschwindigkeit und Automatisierung.

- Bots werden von Kund*innen genutzt, um das Informationsmanagement zu erleichtern.

- Aus der Interaktion mit dem Kunden/der Kundin generierte Daten bilden einen Schlüssel zu den Kundenbedürfnissen.

- Kund*innen geben ihre Daten nur gegen einen Mehrwert preis.

- Unternehmen sind auf intelligente Systeme zur Generierung dieser Daten angewiesen.

- Kund*innen erwarten eine Intelligenz im Unternehmensdialog.

- Der Kundendialog 2025 ist ein Dialog zwischen den Bots des Kunden/ der Kundin und denen des Unternehmens.

... führen zu diesen sechs zentralen Strategieempfehlungen:

- Kundensegmentierungen sind out. Die Einzigartigkeit des Einzelnen steht im Vordergrund.

- Eine radikale Vereinfachung der Kundenkommunikation ist erforderlich.

- Kund*innen sollen situativ wählen können, über welchen Kanal sie kommunizieren wollen.

- Der Einsatz von Personal und Infrastruktur muss einen individuellen und adaptiven Kundendialog ermöglichen.

- Der Kundendialog ist der zentrale Treiber der gesamten Wertschöpfungskette.

- Insourcing statt Outsourcing. Der Kundendialog wird zum Profitcenter des Unternehmens.

$\mathrm{Zu}$ ähnlichen Erkenntnissen kommt Bain (Bain \& Company 2019), wenn auf die Schlussfolgerungen künftiger Kundenerfahrungen hingewiesen wird. Kund*innen wollen demnach an eine einfache Technik herangeführt werden. Die Struktur der Bankstellen muss konsequent in Richtung Digitalisierung ausgerichtet werden. Die Spanne reicht hierbei von vollautomatisierten Service-Stellen bis hin zu beratungsintensiven (durch Technik unterstützten) Vertrauensgeschäften. Berater*innen/Betreuer*innen in Banken/Sparkassen agieren hierbei beziehungsorientiert als Digital-, aber auch Finanzcoaches. Dabei nutzen sie nach innen und außen alle virtuellen Kanäle, die möglich und sinnvoll sind. 


\section{Praxisbeispiel}

Immer mehr digital affine Kund*innen suchen digital affine Bankmitarbeiter*innen als gleichwertige Sparringspartner*innen. Das bedeutet, dass die digitale Kompetenz bei den Mitarbeiter*innen, vor allem am Markt, stark ausgeprägt sein muss. Will ein Haus alle Mitarbeiter*innen in ihrer digitalen Kompetenz stärken und entwickeln, braucht dies Zeit und eine hohe Qualifizierungsund Trainings-/Coachingintensität (vgl. Lern- und Entwicklungsstufen in Abb. 2.2).

Hinzu kommt, dass für die Umsetzung digitaler Dienstleistungen eine entsprechende Infrastruktur bereitgestellt werden muss. Nach wie vor sind zahlreiche Filialen nicht mit schnellen Internetzugängen ausgestattet. Auch fehlen z. B. Tablets, mittels derer dem Kunden/der Kundin flexibler das Internetangebot der Bank/Sparkasse nähergebracht werden kann. Dies führt wiederum dazu, dass erlerntes digitales Wissen der Mitarbeiter*innen in der Praxis (Lernen am Arbeitsplatz) mangels nicht vorhandener Nutzungsmöglichkeiten der Technik nicht angewendet werden kann und wieder verloren geht.

Führungskräfte sollten einerseits, wie bereits angesprochen, die notwendige Nähe für die Begleitung der Mitarbeiter*innen bei der Entwicklung der digitalen Kompetenz gewährleisten. Andererseits liegt es in ihrer Verantwortlichkeit, die für die Arbeit der Mitarbeiter*innen erforderlichen Ressourcen und Mittel sicherzustellen. In der Praxis ein häufiges Dilemma, denn gerade Führungskräfte auf der operativen Ebene verfügen oft nicht über die notwendigen Entscheidungskompetenzen bzw. den Einfluss, um entgegen vorhandener rigider Einsparprogramme Investitionen anzustoßen. Sie baden es am Ende aus, wenn diese strukturellen Aspekte auf der Managementebene bewusst oder unbewusst keine Berücksichtigung bei der Ausrichtung der Strategie finden.

\section{Wertewelten in Verbindung mit Arbeit 4.0}

In seiner Studie „Wertewelten Arbeiten 4.0“ hat das Bundesministerium für Arbeit und Soziales in Zusammenarbeit mit Nextpractice 1.200 Personen danach befragt, wie sie Arbeit heute erleben und welche Arbeitswelt sie sich in der Zukunft, bezogen auf das Jahr 2030 wünschen (Nextpractice 2016).

Die Studie identifiziert sieben klar unterscheidbare Wertewelten:

1. Sorgenfrei von der Arbeit leben können (30\% der Befragten).

2. Den Wohlstand hart erarbeiten (15\%). 
3. Balance zwischen Arbeit und Leben finden (14\%).

4. Sinn außerhalb seiner Arbeit suchen (13\%).

5. Engagiert Höchstleistung erzielen (11\%).

6. Sich in der Arbeit selbst verwirklichen (10\%).

7. In einer starken Solidargemeinschaft arbeiten (9\%).

Da die erste Wertewelt die stärkste Gruppe bildet, soll diese Einschätzung in Tab. 2.3 etwas näher beleuchtet werden. Sie zeigt, welche Themen die befragten Beschäftigten mit Blick auf das Jahr 2030 anstreben und ablehnen.

Aus den bisherigen Ausführungen lässt sich leicht ableiten, dass ausgerechnet die in der Tab. 2.3 aufgeführten ersten vier Aspekte der angestrebten und abgelehn-

Tab. 2.3 Die Top 10 der angestrebten und abgelehnten Themen der Gruppe ,Sorgenfrei von der Arbeit leben können“ und deren Ausprägung in Bezug auf die Entwicklung der Arbeit (Mehrfachnennungen möglich)

\begin{tabular}{l|l|l|l}
\hline Thema (angestrebt) & $\begin{array}{l}\text { Häufigkeit } \\
\text { der Nennung }\end{array}$ & Thema (abgelehnt) & $\begin{array}{l}\text { Häufigkeit } \\
\text { der Nennung }\end{array}$ \\
\hline $\begin{array}{l}\text { Lebensgefühl ist geprägt } \\
\text { von sozialer Sicherheit }\end{array}$ & $25,6 \%$ & $\begin{array}{l}\text { Klima von Druck und } \\
\text { sozialer Kälte bei der Arbeit }\end{array}$ & $28,5 \%$ \\
\hline $\begin{array}{l}\text { Personalschlüssel } \\
\text { ermöglicht arbeiten ohne } \\
\text { Druck }\end{array}$ & $24,9 \%$ & $\begin{array}{l}\text { Trotz sinkendem } \\
\text { Einkommen immer mehr } \\
\text { arbeiten }\end{array}$ & $24,9 \%$ \\
\hline $\begin{array}{l}\text { Planungssicherheit über das } \\
\text { ganze Erwerbsleben }\end{array}$ & $21,3 \%$ & $\begin{array}{l}\text { Berufliche Unsicherheit } \\
\text { erschwert Lebensplanung }\end{array}$ & $24,5 \%$ \\
\hline $\begin{array}{l}\text { In familiärer } \\
\text { Arbeitsatmosphäre } \\
\text { aufgehoben sein }\end{array}$ & $21,3 \%$ & $\begin{array}{l}\text { Arbeitsverdichtung längst } \\
\text { am Limit angekommen. }\end{array}$ & $23,1 \%$ \\
\hline $\begin{array}{l}\text { Beschäftigungslage eröffnet } \\
\text { Chancen für alle }\end{array}$ & $20,6 \%$ & $\begin{array}{l}\text { Der Arbeitsmarkt stellt viel } \\
\text { zu hohe Anforderungen }\end{array}$ & $22,4 \%$ \\
\hline $\begin{array}{l}\text { Entlohnung ist sozial } \\
\text { gerecht und ausreichend }\end{array}$ & $19,9 \%$ & $\begin{array}{l}\text { Eine egoistische und } \\
\text { oberflächliche Gesellschaft }\end{array}$ & $19,5 \%$ \\
\hline $\begin{array}{l}\text { Unternehmer sind } \\
\text { fürsorglich und } \\
\text { rücksichtsvoll }\end{array}$ & $19,1 \%$ & $\begin{array}{l}\text { Dem rücksichtslosen } \\
\text { Konkurrenzkampf } \\
\text { aussetzen }\end{array}$ & $17,3 \%$ \\
\hline $\begin{array}{l}\text { Für soziale Gerechtigkeit } \\
\text { und Umverteilung stehen }\end{array}$ & $19,1 \%$ & $\begin{array}{l}\text { Gesellschaftliche Spaltung } \\
\text { in Arme und Reiche }\end{array}$ & $17,3 \%$ \\
\hline $\begin{array}{l}\text { Kann sich und die Familie } \\
\text { von der Arbeit ernähren }\end{array}$ & $18,4 \%$ & $\begin{array}{l}\text { Umfassende } \\
\text { Flexibilisierung erhöht } \\
\text { Stressniveau }\end{array}$ & $\begin{array}{l}\text { Distanzierte Anonymität: } \\
\text { Jeder ist ersetzbar }\end{array}$ \\
\hline $\begin{array}{l}\text { Hafriedenheit wichtiger als } \\
\text { Karriere }\end{array}$ & $17,0 \%$ & $16,2 \%$ \\
\hline
\end{tabular}


ten Themen aktuell, und in den kommenden Jahren wahrscheinlich noch stärker, mit umgekehrter Wirkung zutreffen werden. Zahlreiche langjährige Mitarbeiter*innen, die nicht auf unmittelbare Vergleichsmöglichkeiten mit anderen Unternehmen oder Arbeitsweisen zurückgreifen können, vergleichen daher den aktuellen Zustand mit dem zurückliegender Jahre und kommen zu einem für sie negativen Ergebnis.

Treffen ihre Wertevorstellungen auf eine sich wandelnde, abweichende Wertewelt der Bank/Sparkasse, entsteht Unzufriedenheit. Auf Dauer leidet die Identifikation und in letzter Konsequenz die Bindung.

Für Führungskräfte ist es daher erforderlich, in Mitarbeitergesprächen oder Teamrunden mit den Wertvorstellungen der Mitarbeiter*innen in Resonanz zu gehen, um diese mit denen des Unternehmens abzugleichen. Weichen beide voneinander ab, beginnt ein Aushandlungsprozess. An dessen Ende lässt sich die/der Mitarbeiter*in (unter Umständen im Zuge von Kompromissen) im Idealfall auf die Wertewelt des Hauses ein. Entsteht keine Einigung kann es für beide Parteien sinnvoll sein, wenn die/der Mitarbeiter*in das Haus verlässt. Nichts zu tun, ist die schlechteste aller Alternativen. Denn stellt sich die Führungskraft dieser Diskussion nicht, kann dies bei der/ dem Mitarbeiter*in eine „Dienst nach Vorschrift-Haltung“ hervorrufen.

\section{Fazit aus diesem Abschnitt}

Es ist unabdingbar, sich als Führungskraft gemeinsam mit den Mitarbeiter*innen mit den veränderten Kundenanforderungen auseinanderzusetzen. In Dienstleistungsunternehmen ist es zwingend erforderlich, das tägliche Geschäft vom Kunden her zu denken. Wer auf Augenhöhe mit Kund*innen partnerschaftlich agieren möchte, muss seine Kompetenzen entsprechend darauf ausrichten.

Bestehen dabei bei Mitarbeiter*innen unterschiedliche Wertvorstellungen, so ist Führung gut darin beraten, sich mit diesen auseinanderzusetzen, um sie mit denen des Unternehmens in Einklang zu bringen und gangbare Wege im Sinne des Unternehmens möglich zu machen.

\subsection{Digitalisierung und Innovationen}

Diese beiden Themen dominieren die aktuelle Geschäftspolitik von Banken und Sparkassen neben der Regulatorik wohl am stärksten. So stand auch der Handelsblatt-Banken-Gipfel in den Jahren 2018/2019 unter dem Fokus der Digitalen Transformation.

Kurz zusammengefasst die Trends und ihre Auswirkungen auf Banken, die im Fokus der Big 4 der Managementberatung (Deloitte, EY, KPMG und PwC) bzw. 
der Big 5 der Strategieberatung (Bain, BCG, McKinsey, Roland Berger und Strategy\&) stehen:

- Artificial Intelligence: Hierdurch gewinnen Finanzinstitute neue Erkenntnisse aus vorhandenen Daten, verbessern die Kundenerfahrung (Customer Experience) und automatisieren Geschäftsprozesse, die bis dato nur mit manuellen Eingriffen funktionierten (Auge-Dickhut und Zerndt 2018).

- Internet of Everything: Finanzinstitute können die Möglichkeiten flexibler Finanzierungsmodelle besser nutzen. So z. B. bei Borrowing-Base-Finanzierungen, bei denen dem Kreditnehmer*in im Rahmen der Finanzierung seines Umlaufvermögens revolvierend liquide Mittel zur Verfügung gestellt werden (Jünemann 2018).

- Virtual und Augmented Reality: Finanzinstitute können es Kund*innen durch computergestützte 3D-Umgebungen, z. B. im Bereich der Immobilienfinanzierung ermöglichen, virtuelle Erlebnisse von Immobilien zu schaffen und eine direkte Schnittstelle zum Berater/zur Beraterin zu generieren (Galer 2017).

- Robotic Process Automatation: Im Bankgeschäft kann eine Anwendung dieser Technologie zu enormen Effizienzsteigerungen bei den etablierten Prozessen führen (Safar 2019).

- Digital Payments: Finanzinstitute können Bezahlsysteme für Kund*innen bequemer und einfacher gestalten (Walter 2018).

- Cyber Security: Finanzinstituten gelingt es, Cyberangriffe mit entsprechenden Softwarelösungen abzuwehren (IBM 2019).

- Cloud Computing: Finanzinstitute lagern Leistungen wie Zahlungsdienstleistungen und Mobile Banking an Cloud-Anbieter aus (PwC 2019).

Die Folgen daraus (Candylabs 2018):

- Neue Technologietrends wachsen exponentiell,

- Plattformen drängen sich zwischen Hersteller und Kund*innen,

- disruptive Angreifer zerstören Märkte immer schneller und

- innovative Geschäftsmodelle verdrängen alte Wertschöpfungsmethoden.

Eine Studie mehrerer renommierter Universitäten kommt mit Blick auf die sich verändernde Rolle der Bankmitarbeitenden zum Schluss: „The banking client 2025 is empowered by digital bots and digitally assisted client advisors.“ (Gasser et al. 2017). 
Es scheint somit bereits in der nahen Zukunft keine Funktion oder Stelle in Banken und Sparkassen mehr zu geben, die nicht maßgeblich vom Einfluss der Digitalisierung betroffen sind.

\section{Fragen}

Was aber wiederum löst diese Entwicklung bei den Mitarbeiter*innen, insbesondere von Finanzinstituten mit traditionellen Geschäftsmodellen, aus?

Abb. 2.4 stellt die Betroffenheit von Mitarbeiter*innen bei betrieblichen Veränderungen und mögliche Veränderungsauslöser gegenüber.

Aus der Perspektive der einzelnen Mitarbeitenden ergeben sich zwei Blickrichtungen auf anstehende Veränderungen (siehe Betroffenheits-Matrix in Abb. 2.4):

- Wie umfangreich ist die Veränderung und wie sehr beeinflusst diese das Denken und Verhalten?

- In welchem Umfang wird die Veränderung als persönliche Bedrohung (Arbeitsplatz, Stellung, Reputation, etc.) empfunden?

Sind das Ausmaß der Veränderungen und die wahrgenommene, persönliche Be-
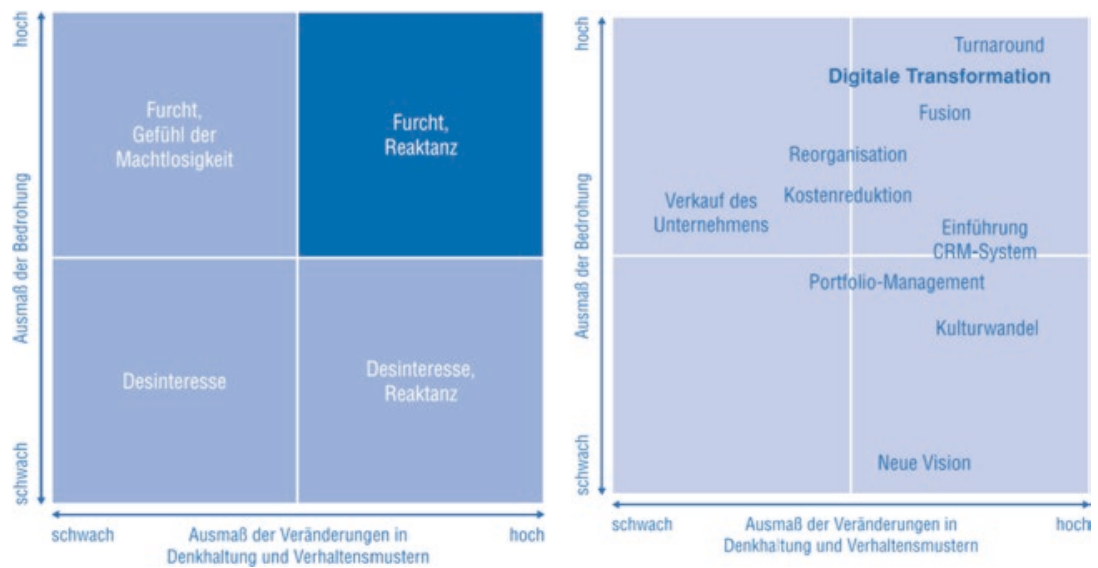

Abb. 2.4 Change-Auslöser und Matrix der Betroffenheit

drohung hoch ausgeprägt, reagieren Menschen mit Angst und Reaktanz. In Folge darauf verschließen sie sich der Veränderung und/oder versuchen den ursprünglichen 
Grad der persönlichen Freiheit, den sie vor der Veränderung für sich wahrgenommen haben, wiederherzustellen.

Ordnet man nun mögliche anstehende Veränderungen in diese BetroffenheitsMatrix ein, so findet sich ausgerechnet die Digitale Transformation im kritischsten Sektor (Kreutzer et al. 2017).

\section{Praxisbeispiel}

In einem Change-Workshop mit Servicekräften einer lokalen Bank sollten die Mitarbeiter*innen bildhaft ihre Vorstellungen zur Filiale der Zukunft festhalten. Das Ergebnis zeigte:

- Weniger Filialen als aktuell vorhanden,

- kaum noch Mitarbeiter*innen im Servicebereich,

- einen hohen Grad an Automatisierung,

- eine stärkere Konzentration auf Beratung.

Die Beschäftigten schätzten somit die mögliche Entwicklung ihres Aufgabenbereichs richtig ein.

Damit verbunden ging, vor allem bei den älteren Mitarbeiter*innen, eine große Verunsicherung und Sorge um die eigene Entwicklung bzw. den künftigen Einsatzbereich einher.

Die Kernfragen, die sie sich stellten, waren:

- Werde ich in naher Zukunft die Filiale wechseln müssen?

- Wird es meinen Aufgabenbereich überhaupt noch geben?

- Muss ich künftig, obwohl ich mich all die Jahre dagegen entschieden habe, in der Beratung aktiv sein?

- Wie schnell muss ich mich an die neue Situation gewöhnen?

- Schaffe ich es überhaupt, in meinem Alter den Anforderungen noch gerecht zu werden?

In den Vorgesprächen zu den Workshops vermutete die Vertriebsleitung des Hauses eine grundsätzliche starke Abwehrhaltung gegenüber den anstehenden Neuerungen. Stattdessen zeigten sich eher Verunsicherung und Angst.

Der Vorstand der Bank hatte einen klaren Ausblick auf künftige Entwicklungen vermittelt. Die direkten Führungskräfte hatten es jedoch bis zu diesem Zeitpunkt versäumt, die betroffenen Mitarbeiter*innen abzuholen. 


\section{Fazit aus diesem Abschnitt}

Führung soll Orientierung schaffen und Sinn stiften, statt Angst und Perspektivlosigkeit einen Nährboden zu geben. Worthülsen und distanziertes Führungsverhalten lösen eher letzteres aus. Eine ehrliche und faire Auseinandersetzung mit den Zukunftsthemen der Bank/Sparkasse schafft Klarheit und mehr Verbindlichkeit im Zuge der zu gehenden Entwicklungsschritte bei den Mitarbeiter*innen.

\subsection{Folgen für Banken und Sparkassen}

Welche Auswirkungen haben diese Rahmenbedingungen für Banken und Sparkassen?

Oliver Wyman (2018) entwickelt zwei Szenarien:

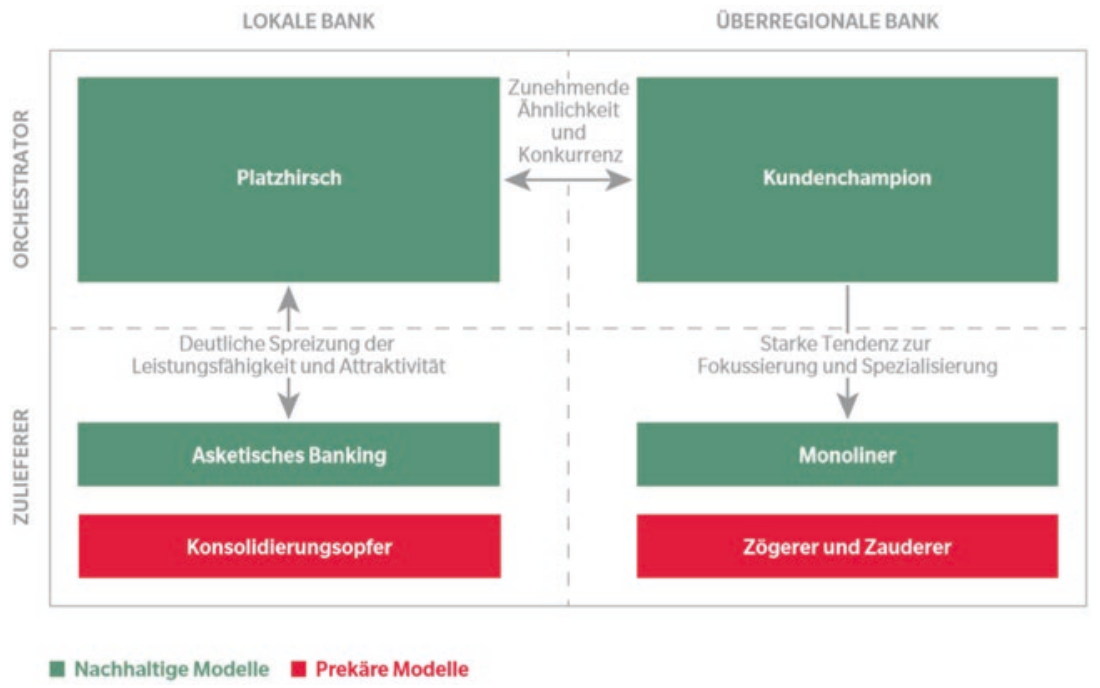

Abb. 2.5 Evolutionsszenario: Nachhaltige und prekäre Positionierung von lokalen und überregionalen Banken als Orchestratoren bzw. Zulieferer (Oliver Wyman 2018) 


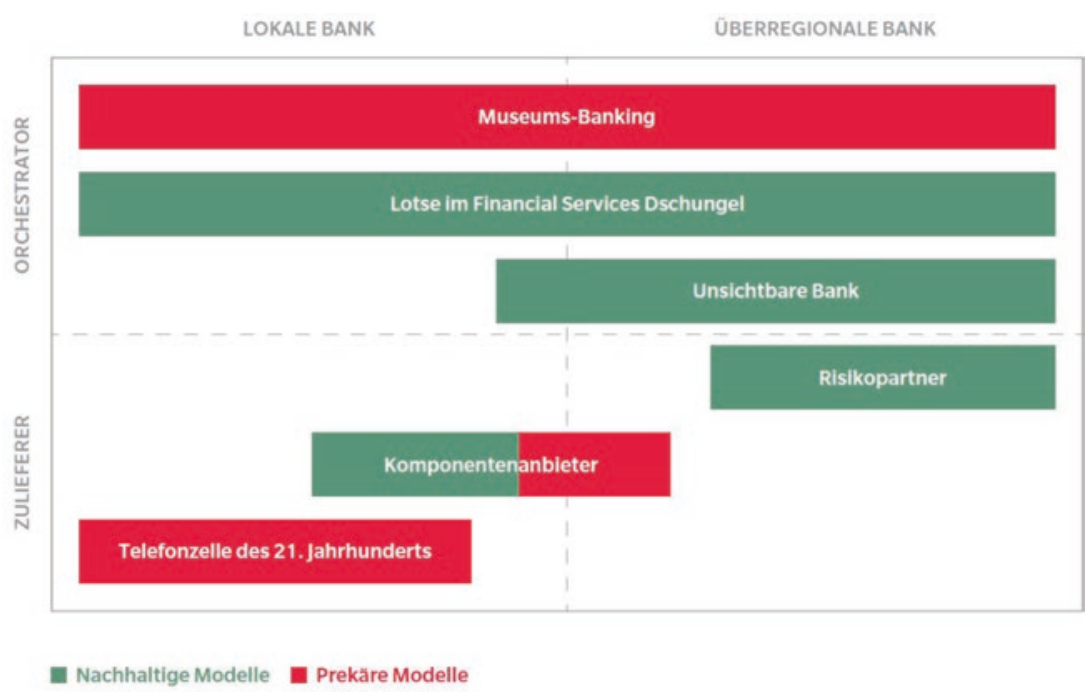

Abb. 2.6 Disruptionsszenario: Nachhaltige und prekäre Positionierung von Banken als Orchestratoren bzw. Zulieferer (Oliver Wyman 2018)

- Ein Evolutionsszenario (vgl. Abb. 2.5), in dem die Stabilität des deutschen Bankenmarktes den Marktteilnehmer*innen eine schrittweise Anpassung an die Veränderungen erlaubt.

- Ein Disruptionsszenario (vgl. Abb. 2.6), bei dem insbesondere umfassende Veränderungen durch neue Technologien zu drastischen Folgen am Arbeitsplatz und bei den Arbeitsprozessen führen.

Auch wenn Banken und Sparkassen aktuell vereinzelt das Regionalprinzip aufgeben, die Mehrheit agiert in einem lokalen Markt. Um sich dort im Zuge einer schrittweisen Anpassung (Evolutionsszenario) bis zum Jahr 2030 als „Platzhirsche“" zu behaupten, müssen sie wie folgt auftreten:

- Als auf die lokale Wirtschaft und deren Bedürfnisse angepasster Allfinanzanbieter,

- mit einer intelligenten Nutzung von überregionalen (durch technologische Plattformen gestützten) Angeboten,

- mit einer starken Eigenmarke,

- der Verbindung zu anderen lokalen Services und 
- einem starken Engagement in der lokalen Community, vor allem in Ballungsräumen und wirtschaftlich starken Regionen.

Anders stellt sich dies bei einer disruptiven Entwicklung dar, die einschneidendere Veränderungen nach sich zieht.

Führen Finanzinstitute ihr Geschäftsmodell als traditioneller Vollanbieter mit Abdeckung der gesamten Wertschöpfungskette fort, so wird in diesem Szenario ihr Modell als prekär und damit nicht überlebensfähig angesehen.

Für Banken und Sparkassen zeichnet sich daher vornehmlich eine Rolle als Lotse im Financial Services Dschungel ab. Als Orchestratoren und Zulieferer fungieren sie hierbei

- als Bindeglied zwischen Technologie und Mensch,

- mit der Aufgabe, Betreuungs-, Erklärungs- und Maklertätigkeiten umzusetzen und

- als Filterfunktion zwischen zahlreichen Angeboten und Kund*innen.

Ungeachtet eines möglichen evolutionären oder disruptiven Szenarios sieht Oliver Wyman den deutschen Bankenmarkt reduziert. Unter Einbindung der Szenarien ergibt sich eine Verringerung von heute 1.600 Instituten auf ca. 300 bis $150 \mathrm{im}$ Jahr 2030.

Ob es größeren Unternehmen, auch im Zuge der vorgenannt prognostizierten anstehenden Konsolidierungswelle, besser gelingt, Führung systematisch zu etablieren? Das folgende Kapitel versucht Antworten hierauf, als auch zu weiteren Fragen rund um strategische, kulturelle und organisatorische Rahmenbedingungen zu geben.

\section{Reflexionsfragen zum Kapitel}

- Handelt und kommuniziert der Gesamtvorstand der Bank/Sparkasse abgestimmt und geschlossen?

- Werden Stellvertreterkriege auf den Ebenen unterhalb des Vorstandes vermieden, indem strategische Ziel-Inkongruenzen auf der Ebene des Managements frühzeitig und offen ausdiskutiert und so weit wie möglich ausgeräumt werden?

- Wird das Geschäftsmodell mutig und unter Einbindung des vorhandenen Know-how des Hauses weiterentwickelt? 
- Betrachtet man die Personalkapazitäten frühzeitig und ehrlich unter dem Fokus der in den kommenden Jahren ausscheidenden Generation der Babyboomer? Wie wird der Wissenstransfer von Schlüsselpersonen sichergestellt?

- Wie geht Führung mit den Werten, Einstellungen und Job-Präferenzen der unterschiedlichen Generationen im Team um?

- Wie geht die Bank/Sparkasse bzw. Führung mit den unterschiedlichen Erwartungen der Mitarbeiter*innen an die Arbeitswelt 4.0 um?

- Welche personalpolitischen Instrumente sind den Führungskräften an die Hand gegeben, um junge Mitarbeiter*innen zu binden und die Motivation reifer Mitarbeiter*innen aufrecht zu erhalten?

- Lebt Führung die Umsetzung digitaler Kompetenzen vor? Stellen Führungskräfte sicher, dass Mitarbeiter*innen digitale Kompetenzen an den Kundenkontaktpunkten, dort wo erforderlich, auf Augenhöhe umsetzen?

- Wie können Mitarbeiter*innen Innovationen und der Einsatz von neuen Technologien chancen- und nutzenorientiert vermittelt werden?

- Wie reagieren Führungskräfte auf die Ängste und Fragen zögerlicher Mitarbeiter*innen? Gibt man ihnen ausreichend Zeit und Möglichkeiten für die geforderten Entwicklungsschritte?

\section{Literatur}

2b AHEAD ThinkTank (2018) Kundendialog 2025. Der Dialog zwischen intelligenten Systemen. http://www.zukunft.business/foresight/trendstudien/. Zugegriffen am 09.11.2019

Agentur Junges Herz (2019) Generation Z: Was erwartet den Arbeitsmarkt? https://www. agentur-jungesherz.de/generation-z/. Zugegriffen am 28.11.2019

Auge-Dickhut S, Zerndt T (2018) Künstliche Intelligenz - Mehr als Bankroboter. https:// www.handelszeitung.ch/kuenstliche-intelligenz-mehr-als-bankroboter. Zugegriffen am 10.11.2019

Bain \& Company (2019) Reimagining the digital bank branch of the future: let's get practical. https://www.bain.com/insights/reimagining-the-digital-branch-of-the-future-lets-get-practical/. Zugegriffen am 18.02.2019

Buch CM (2018) Deutsche Bundesbank. https://www.bundesbank.de/resource/blob/758264/ 1eed3dbb51eba9e0da8420e6a1fb33d9/mL/2018-09-03-buch-download.pdf. Zugegriffen am 21.03.2019

Burkhart A (2012) Rollenkonflikt und Rollenstress bei Führungskräften. HR Consult Rev 1:24-28

Candylabs (2018) Virtual Prototyping - Wie Produktideen ohne Prototypen im Markt getestet werden können. https://www.candylabs.de/dokumente/108/Whitepaper_Virtual-Prototyping.pdf. Zugegriffen am 09.11.2019

Felfe J (2012) Demografischer Wandel in der Bankenbranche. Helmut-SchmidtUniversität, Hamburg 
Galer S (2017) Banken entdecken neue Welt des Kundenkomforts mit Virtual und Augmented Reality. https://news.sap.com/germany/2017/11/banken-augmented-reality/. Zugegriffen am 10.11.2019

Gasser U, Gassmann O, Hens T, Leifer L, Puschmann T, Zhao L (2017) Digital Banking 2025. https://www.alexandria.unisg.ch/publications/253962. Zugegriffen am 10.11.2019

Hülsbeck M, Scheren S (2017) Generationsspezifische Arbeitswerte, Mitarbeiterbindung und HRM. In: Jochmann W et al (Hrsg) HR-Exzellenz. Springer Fachmedien, Wiesbaden

IBM (2019) Geschäftserfolg braucht Sicherheit. https://www.ibm.com/industries/de-de/ campaign/banking-security-risk-compliance. Zugegriffen am 10.11.2019

Jünemann MD (2018) Der Bank Blog - Das Internet der Dinge ermöglicht neue Finanzierungswege. https:/www.der-bank-blog.de/das-internet-dinge-2/firmenkunden/31558/. Zugegriffen am 10.11.2019

Kreutzer RT, Neugebauer T, Pattloch A (2017) Digital business leadership. Springer Gabler, Wiesbaden

Krüger KH (2016) Gesellschaftlicher Wertewandel: Generation X, Y, Z - und dann? In: Klaus H, Schneider HJ (Hrsg) Personalperspektiven. Springer Fachmedien, Wiesbaden

Nextpractice, Bundesminiterium für Arbeit und Soziales (2016) Studie „Wertewelten Arbeiten 4.0“. https://www.bmas.de/SharedDocs/Downloads/DE/PDF-Publikationen/Forschungsberichte/wertewelten-arbeiten-vier-null.pdf;jsessionid=AA156385D33E257A2C6451CC6 358E02C?_blob=publicationFile\&v=2. Zugegriffen am 09.11.2019

Oliver Wyman (2018) Bankenreport Deutschland 2030. https://www.oliverwyman.de/ our-expertise/insights/2018/feb/Bankenreport-Deutschland-2030.html. Zugegriffen am 09.11.2019

Priewasser E (1994) Die Priewasser Prognose. Fritz Knapp, Frankfurt

PwC Cloud Computing im Bankensektor (2018) https://www.pwc.de/de/finanzdienstleistungen/pwc-cloud-computing-im-bankensektor.pdf. Zugegriffen am 02.03.2019

Safar M (2019) Wie Banken von Robotic Process Automation (RPA) profitieren können. https://weissenberg-solutions.de/wie-banken-von-robotic-process-automation-rpa-profitieren-koennen/. Zugegriffen am 02.03.2019

Spektrum.de (2019) Lexikon der Psychologie - Feldtheorie. https://www.spektrum.de/lexikon/psychologie/feldtheorie/4894. Zugegriffen am 09.02.2019

TME Institut für Vertrieb und Transformationsmanagement e.V (2014) Innovative Geschäftsmodelle im Banking. Factbook 2015. Bank, Köln

Walter A (2018) Digital payments. https://www.frankfurt-school.de/en/home/newsroom/ news/2018/Dezember/digital-payments. Zugegriffen am 10.11.2019

Wuermeling J (2019) Deutsche Bundesbank, Herausforderungen für den deutschen Bankensektor. https://bundesbank.de/de/presse/reden/herausforderungen-fuer-den-deutschen-bankensektor-767072. Zugegriffen am 09.11.2019

zeb (2018) Private Banking Studie Deutschland 2018. https://www.it-finanzmagazin.de/ zeb-privatkundenstudie-2018-negative-aussichten-fuer-das-deutsche-retail-banking-79458/. Zugegriffen am 13.11.2019 